\title{
Effect of the Sodium-Glucose Cotransporter 2 Inhibitor, Dapagliflozin, on Genitourinary Infection in an Animal Model of Type 2 Diabetes
}

\author{
Jin Bong Choi ${ }^{1}$, Je Mo Yoo ${ }^{2}$, Ye-Jee Lee ${ }^{3}$, Jae Woong Kim${ }^{4}$, Seung-Ju Lee², Hee Youn Kim², Dong Sup Lee ${ }^{2}$, Seung-Hyun Ko ${ }^{5}$, \\ Hyun-Sop Choe ${ }^{2}$ \\ ${ }^{1}$ Department of Urology, Bucheon St. Mary's Hospital, College of Medicine, The Catholic University of Korea, Seoul, Korea \\ ${ }^{2}$ Department of Urology, St. Vincent's Hospital, College of Medicine, The Catholic University of Korea, Seoul, Korea \\ ${ }^{3}$ Division of Endocrinology \& Metabolism, Department of Internal Medicine, Seoul St. Mary's Hospital, College of Medicine, The Catholic University of \\ Korea, Seoul, Korea \\ ${ }^{4}$ Goldman Urology Clinic, Incheon, Korea \\ ${ }^{5}$ Division of Endocrinology \& Metabolism, Department of Internal Medicine, St. Vincent's Hospital, College of Medicine, The Catholic University of Korea,
} Suwon, Korea

Purpose: To investigate the effect of dapagliflozin, a sodium-glucose cotransporter 2 inhibitor, on inflammatory cytokines of urogenital tissue in a rat model of type 2 diabetes (T2DM) to infer pharmaceutical influence of dapagliflozin on genitourinary infection or inflammation.

Methods: Study animals were divided into the following 4 groups of 10 animals each: (1) the Otsuka Long-Evans Tokushima Fatty (OLETF)-DA group treated with dapagliflozin at $1.0 \mathrm{mg} / \mathrm{kg} / \mathrm{day}$, (2) the OLETF-VO group treated with voglibose at 0.6 $\mathrm{mg} / \mathrm{kg} /$ day, (3) the control group (OLETF-CO) given water, and (4) the Long-Evans Tokushima Otsuka (LETO) rats were included as nondiabetic control group. Changes in blood glucose, 24-hour urine volume, and urine glucose were measured. The interleukin-1 (IL-1 $\beta$ ) and interleukin-18 (IL-18) levels in the bladder and the urethra were quantified, respectively.

Results: The urine glucose level and the 24-hour urine volume at 12 weeks of treatment were significantly higher in the OLETF-DA group than that in any other group $(\mathrm{P}<0.05)$. The cytokine analysis of the bladder and urethra showed higher IL18 and IL- $1 \beta$ in the OLETF-DA and the OLETF-CO groups than that in the OLETF-VO and LETO groups $(\mathrm{P}<0.05)$. The cytokine levels did not differ between the OLETF-DA and the OLETF-CO groups, and the level of IL-18 in the OLETF-DA group was higher in the urethra than in the bladder.

Conclusions: This study revealed that dapagliflozin increased the urine glucose concentration, resulting in an inflammatory response remain in the urogenital tract as the untreated diabetic rats. Therefore, when treating patients with T2DM with dapagliflozin, careful attention should be paid to genitourinary infection or inflammation.

Keywords: Diabetes mellitus; Dapagliflozin; Cytokines; Urinary tract infection

- Fund/Grant Support: This study was funded by a grant from the Korean Diabetes Association.

- Research Ethics: The treatment protocol was approved by the Catholic University of Korea Animal Ethics Committee (CUMC-2015-0182) and

handled according to National Institutes of Health guidelines.

- Conflict of Interest: No potential conflict of interest relevant to this article was reported.

Corresponding author: Hyun-Sop Choe (iD https://orcid.org/0000-0001-7387-9595 Department of Urology, St. Vincent's Hospital, College of Medicine, The Catholic University of Korea, 93 Jungbu-daero, Paldal-gu, Suwon 16247, Korea E-mail: hschoe96@gmail.com

Submitted: November 6, 2019 / Accepted after revision: February 11, 2020
This is an Open Access article distributed under the terms of the Creative Commons Attribution Non-Commercial License (https://creativecommons.org/licenses/by-nc/4.0/) which permits unrestricted non-commercial use, distribution, and reproduction in any medium, provided the original work is properly cited. 


\begin{abstract}
- HIGHLIGHTS
- Dapagliflozin increased the urine glucose concentration in a rat model of type 2 diabetes.

- Treatment with dapagliflozin resulted in an inflammatory response remain in the urogenital tract as the untreated diabetic rats.
\end{abstract}

\section{INTRODUCTION}

Infectious diseases, including urinary tract infection (UTI), are more frequent in patients with type 2 diabetes mellitus (T2DM) [1-3]. Hyperglycemia causes immune dysfunctions, including neutrophil dysfunction, disorders of humoral immunity, and impairment of the antioxidant defense system [4]. Diabetic neuropathy may promote bladder dysfunction, resulting in urinary retention and UTI [5]. Among the various mechanisms that contribute to the risk of UTI, higher urine glucose levels may promote the growth of bacteria $[6,7]$.

Sodium-glucose cotransporter 2 (SGLT2), located mainly in the proximal convoluted tubule of the nephron, is responsible for most the of glucose reabsorption in the kidney [8]. Recently, SGLT2 inhibitors have been proposed as a novel therapeutic agent for T2DM by reducing renal glucose reabsorption [9]. Among them, dapagliflozin is a highly selective inhibitor of SGLT2 [10], and many clinical studies have reported its efficacy and safety in patients with T2DM as monotherapy or combination therapy [11]. With the approval of dapagliflozin in the European Union, the U.S. Food and Drug Administration (FDA) approved dapagliflozin on January 2014 for glycemic control, along with diet and exercise, in patients with T2DM. In addition to the glucose-lowering effect, dapagliflozin reduces body weight and blood pressure without hypoglycemia [12].

Interestingly, SGLT2 inhibitor treatment improves hyperglycemia; however, a contrarily high glucose concentration is created in the urinary tract locally. In other words, hyperglycemia is improved systematically, but the glucose concentration is markedly increased in the genitourinary system at the same time. Therefore, the risk of genitourinary infection might increase from the beginning of clinical trials when using SGLT2 inhibitor because bacteria can grow well in a high glucose containing medium. Many clinical studies and meta-analyses also have evaluated the association between genitourinary infection and SGLT2 inhibitors [13-18].

In general, patients with T2DM who are treated with SGLT2 inhibitors showed increased urine output [19]. And previous meta-analysis showed a 4-fold increased risk of genital infection (GI) and 1.5-fold increased risk of UTI in the SGLT2 in- hibitors treatment group compared to the placebo group [20]; however, in a more recent meta-analysis, the risk of UTI was not increased with SGLT2 inhibitor use compared to placebo or active comparator, although dapagliflozin $10 \mathrm{mg}$ daily was associated with a significantly increased risk of UTI compared to placebo [13]. Therefore, controversy remains in the UTI-related safety profile of SGLT2 inhibitors, especially for dapagliflozin, and further analyses are needed to evaluate the potential mechanism of action of dapagliflozin for UTI.

The objective of the present study was to evaluate the effect of dapagliflozin on genitourinary infection in a laboratory using a rat model of T2DM. It is hard to measure genitourinary infections qualitatively and quantitatively because it is difficult to guarantee an aseptic condition in an animal study. Therefore, instead of trying to measure the infection directly, we chose to measure inflammation associated with the infection. Host immune responses are critically important in the antimicrobial defense of the urinary tract, and these cytokines can represent intracorporeal evidence of the infectious process [21]. Inflammatory cytokines, including interleukin-1 $\beta$ (IL-1 $\beta$ ), interleukin-18 (IL-18), and tumor necrosis factor $\alpha$ (TNFa) are known to play an important role in mediating the innate immune response and are involved in the upregulation of inflammatory reactions [22]. Therefore, in this study, we investigated the effect of dapagliflozin on genitourinary infection by measuring tissue inflammatory cytokines in a rat model of T2DM.

\section{MATERIALS AND METHODS}

\section{Experimental Animals and Treatment}

The treatment protocol was approved by the Catholic University of Korea Animal Ethics Committee and handled according to National Institutes of Health guidelines. Six-week-old male Long-Evans Tokushima Otsuka (LETO) rats for the nondiabetic control group and Otsuka Long-Evans Tokushima Fatty (OLETF) rats were purchased from Central Lab Animal incorporation (Seoul, Korea). Male rats were selected to collect sufficient amount of urethral tissue to reflect the infection or inflammation of genital organ. All animals were housed under a $12 / 12$ hours light-dark cycle and a temperature of $22^{\circ} \mathrm{C}$ with ad 
libitum feeding during throughout the experimental period. The OLETF rats are a commonly known rat model of T2DM with characteristic features of hyperinsulinemia, hyperglycemia, insulin resistance, hypertriglyceridemia, and mild obesity [23]. Voglibose, an alpha-glucosidase inhibitor, was used for comparative analysis to dapagliflozin because it reduces glucose absorption across the gastrointestinal tract regardless urinary glucose excretion [24].

The OLETF rats were randomly divided into the following 3 groups of 10 animals each: (1) the OLETF-DA group treated with dapagliflozin at $1.0 \mathrm{mg} / \mathrm{kg} /$ day (AstraZeneca, Mölndal, Sweden), (2) the OLETF-VO group treated with voglibose at 0.6 $\mathrm{mg} / \mathrm{kg} /$ day (Sigma-Aldrich, St. Louis, MO, USA), and (3) the control group (OLETF-CO) given water. The 10 LETO rats that did not have diabetic syndrome were included as nondiabetic control group (LETO group) and given water. In each group, once-daily oral administration of either drug dissolved in drinking water was continued for 12 weeks starting at 14 weeks of age. The dosage and the treatment period of administered dapagliflozin and voglibose were determined based on previous animal studies [25-27].

\section{Urine and Biochemistry Analysis}

To measure the 24-hour urine volume and urine glucose after 12 weeks of treatment, the rats were individually housed in plastic metabolic cages (Tecniplast Gazzada, Buguggiate, Italy). The automated analyzer (Beckman Instruments, Inc., Fullerton, CA, USA) was utilized to determine the urine glucose concentration. The blood glucose concentration was measured via tail snipping after overnight fasting using the Accu-Chek glucometer (Roche, Basel, Switzerland) after 12 weeks of treatment.

\section{Rat Tissue Assays}

After 12 weeks of treatment, the bladder and the urethra were removed from all animals, and all tissues were cryopreserved. The frozen tissue was homogenized following the manufacturer's instructions (Magnetic Luminex Assay multiplex kits, R\&D Systems Inc., Minneapolis, MN, USA). First, $50 \mu \mathrm{L}$ of sample was added per well. An additional $50 \mu \mathrm{L}$ of the diluted microparticle cocktail was added to each well, and the reaction was incubated for 2 hours at room temperature on a shaker at 800 $\mathrm{rpm}$. Next, the beads were washed 3 times, and $50 \mu \mathrm{L}$ of the diluted biotin-antibody cocktail was added to each well. The mixture was covered and incubated for 1 hour at room temperature on a shaker at $800 \mathrm{rpm}$. After repeating this step, $50 \mu \mathrm{L}$ of the diluted streptavidin-phycoerythrin conjugate compound was added and allowed to incubate for 30 minutes. The beads were then washed again and incubated with $100 \mu \mathrm{L}$ of the wash buffer for 2 minutes at room temperature [28,29].

To measure the concentrations of IL-1 $\beta$ and IL-18 in both tissues, the median fluorescence intensity (MFI) of each cytokine was analyzed using the Luminex MAGPIX analyzer $(\mathrm{R} \& \mathrm{D}$ systems) according to the manufacturer's instructions. The duplicate readings for each sample were averaged and the average blank MFI was subtracted. A standard curve for each sample was created by reducing the data using computer software. Finally, the assay was calibrated against highly purified recombinant rat biomarkers produced at R\&D Systems.

\section{Statistical Analysis}

All data are expressed as the mean \pm standard error. To compare the significant differences among groups, 1-way analysis of variance was calculated using $\mathrm{R}$ ver. 3.5.1 ( $\mathrm{R}$ Foundation for Statistical Computing, Vienna, Austria) software. Values of $\mathrm{P}<0.05$ were considered significant.

\section{RESULTS}

\section{Urine and Biochemistry Analysis}

After 12 weeks of treatment, the mean fasting blood glucose

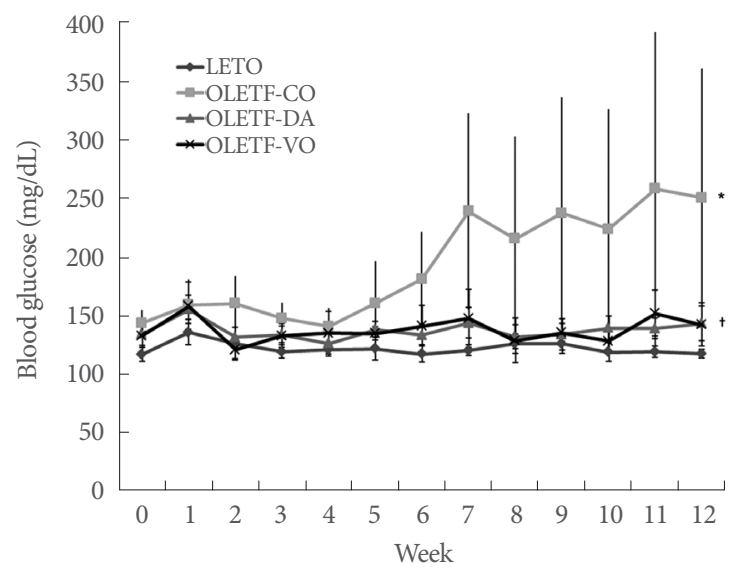

Fig. 1. Changes in blood glucose during the experimental period in OLETF-DA, OLETF-VO, OLETF-CO, and LETO group. LETO, Long-Evans Tokushima Otsuka; OLETF-CO, control group given water; OLETF-DA, group treated with dapagliflozin at $1.0 \mathrm{mg} / \mathrm{kg} /$ day; OLETF-VO, group treated with voglibose at $0.6 \mathrm{mg} / \mathrm{kg} /$ day. ${ }^{*} \mathrm{P}<0.05$, OLETF-CO vs. other groups; ${ }^{\dagger} \mathrm{P}<0.05$, OLETF-DA or OLETF-VO vs. LETO. Values are expressed as the mean \pm standard error. 
levels in the OLETF-CO group $(168.6 \pm 17.9 \mathrm{mg} / \mathrm{dL})$ were significantly higher than those in any other group $(\mathrm{P}<0.05)$. The OLETF-DA group $(116.6 \pm 1.7 \mathrm{mg} / \mathrm{dL})$ and the OLETF-VO group $(123.3 \pm 6.2 \mathrm{mg} / \mathrm{dL})$ showed higher fasting glucose levels than those of the LETO group $(94.7 \pm 1.2 \mathrm{mg} / \mathrm{dL})(\mathrm{P}<0.05)$ (Fig. 1).

The urine glucose level at 12 weeks of treatment was significantly higher in the OLETF-DA group $(9,100.0 \pm 443.2 \mathrm{mg} / \mathrm{dL})$ than that in any other group (LETO group, $50.7 \pm 11.2 \mathrm{mg} / \mathrm{dL}$; OLETF-CO group, $64.3 \pm 11.0 \mathrm{mg} / \mathrm{dL}$; OLETF-VO group,

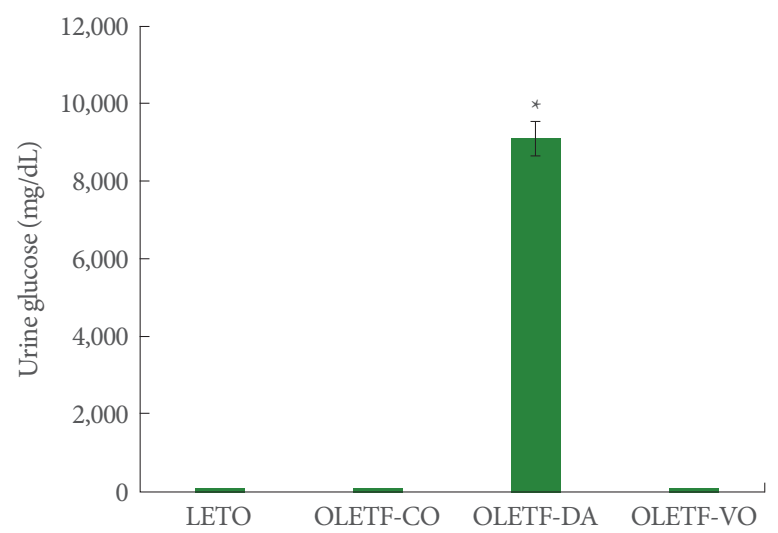

Fig. 2. The urine glucose level at 12 weeks of treatment. LETO, Long-Evans Tokushima Otsuka; OLETF-CO, control group given water; OLETF-DA, group treated with dapagliflozin at 1.0 $\mathrm{mg} / \mathrm{kg} /$ day; OLETF-VO, group treated with voglibose at 0.6 $\mathrm{mg} / \mathrm{kg} /$ day. Values are expressed as the mean \pm standard error. OLETF-DA, group treated with dapagliflozin at $1.0 \mathrm{mg} / \mathrm{kg} / \mathrm{day}$. ${ }^{\star} \mathrm{P}<0.05$, OLETF-DA vs. other groups.
$31.5 \pm 9.3 \mathrm{mg} / \mathrm{dL})(\mathrm{P}<0.05)$ (Fig. 2). The 24-hour urine volume was also significantly higher in the OLETF-DA group (24.6 \pm $5.4 \mathrm{~mL}$ ) compared to that of the other groups (LETO group, $8.9 \pm 3.3 \mathrm{~mL}$; OLETF-CO group, $11.6 \pm 4.1 \mathrm{~mL}$; OLETF-VO group, $12.5 \pm 3.1 \mathrm{~mL})(\mathrm{P}<0.05)$.

\section{Rat Tissue Biochemical Assays}

We quantified the IL-1 $\beta$ and IL-18 levels in the bladder and the urethra, respectively.

The mean concentration of IL-18 in the bladder was significantly higher in the OLETF-DA and the OLETF-CO groups $(547.7 \pm 153.2 \mathrm{pg} / \mathrm{mL}$ and $509.9 \pm 136.3 \mathrm{pg} / \mathrm{mL}$, respectively) than that in the OLETF-VO and LETO groups $(113.1 \pm 48.3 \mathrm{pg} /$ $\mathrm{mL}$ and $127.5 \pm 34.2 \mathrm{pg} / \mathrm{mL}$, respectively) $(\mathrm{P}<0.05)$. The IL-18 concentrations in the OLETF-DA and the OLETF-CO groups were lower in the bladder $(547.7 \pm 153.2 \mathrm{pg} / \mathrm{mL}$ and $509.9 \pm$ $136.3 \mathrm{pg} / \mathrm{mL}$, respectively) than that in the urethra $(1,061.9 \pm$ $556.8 \mathrm{pg} / \mathrm{mL}$ and $1,130.4 \pm 597.1 \mathrm{pg} / \mathrm{mL}$, respectively). No differences in the IL-18 levels in either the bladder or the urethra between the OLETF-DA and the OLETF-CO groups were detected ( $\mathrm{P}>0.05$ each) (Fig. 3).

The mean concentration of IL- $1 \beta$ in the bladder was higher in the OLETF-DA and the OLETF-CO groups $(55.2 \pm 23.1 \mathrm{pg} /$ $\mathrm{mL}$ and $63.9 \pm 29.9 \mathrm{pg} / \mathrm{mL}$, respectively) than that in the OLETF-VO and LETO groups $(23.5 \pm 3.6 \mathrm{pg} / \mathrm{mL}$ and $31.3 \pm 6.6$ $\mathrm{pg} / \mathrm{mL}$, respectively). No statistically significant difference between the OLETF-DA and the OLETF-CO groups was detected ( $\mathrm{P}>0.05$ each) (Fig. 4).
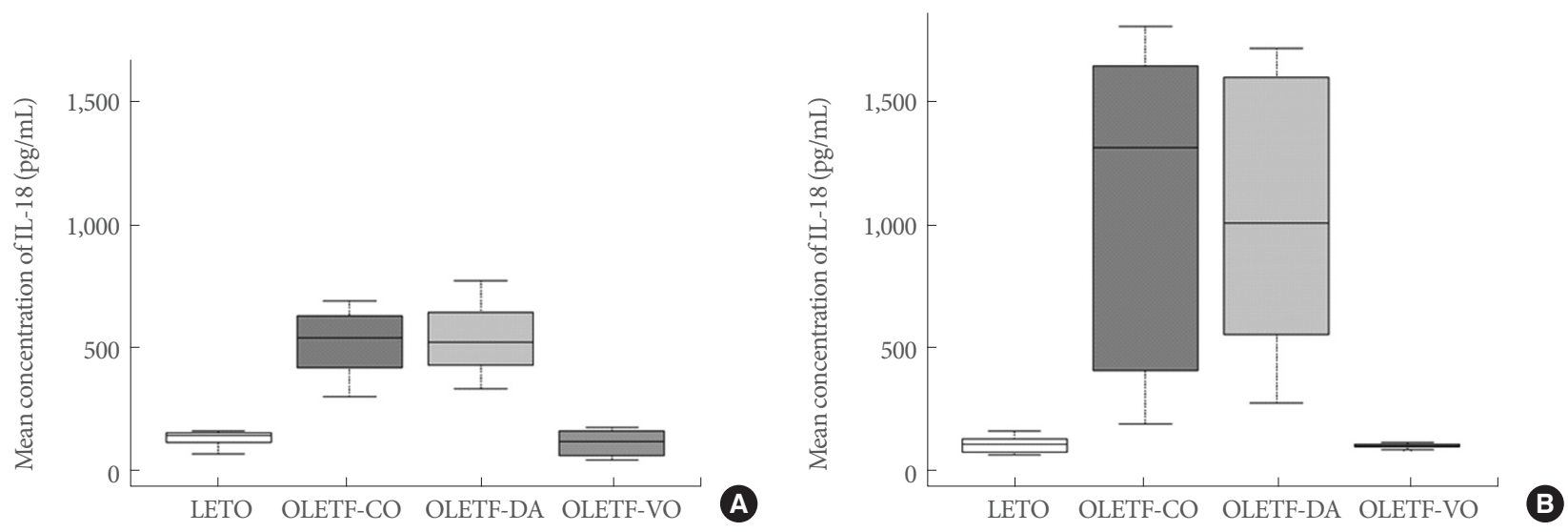

Fig. 3. The mean concentration of IL-18 in the bladder (A) and the urethra (B) at 12 weeks of treatment. LETO, Long-Evans Tokushima Otsuka; OLETF-CO, control group given water; OLETF-DA, group treated with dapagliflozin at $1.0 \mathrm{mg} / \mathrm{kg} / \mathrm{day}$; OLETF-VO, group treated with voglibose at $0.6 \mathrm{mg} / \mathrm{kg} /$ day. $\mathrm{P}<0.05$, OLETF-DA and OLETF-CO vs. OLETF-VO and LETO; P $>0.05$, OLETF-DA vs. OLETF-CO. Values are expressed as the mean \pm standard error. 

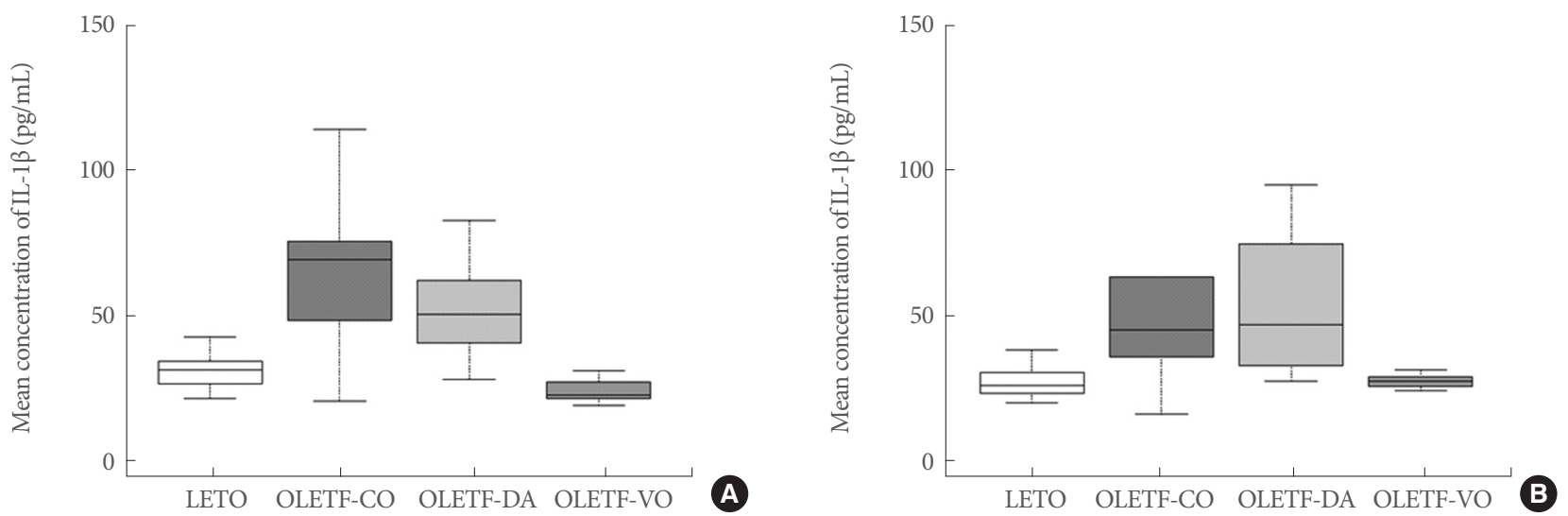

Fig. 4. The mean concentration of interleukin (IL)-1 $\beta$ in the bladder (A) and the urethra (B) at 12 weeks of treatment. LETO, LongEvans Tokushima Otsuka; OLETF-CO, control group given water; OLETF-DA, group treated with dapagliflozin at $1.0 \mathrm{mg} / \mathrm{kg} / \mathrm{day}$; OLETF-VO, group treated with voglibose at $0.6 \mathrm{mg} / \mathrm{kg} /$ day. $\mathrm{P}<0.05$, OLETF-DA and OLETF-CO vs. OLETF-VO and LETO; $\mathrm{P}>0.05$, OLETF-DA vs. OLETF-CO. Values are expressed as the mean \pm standard error.

\section{DISCUSSION}

The main findings of this preclinical study are as follows: (1) the 24-hour urine glucose was significantly higher in the dapagliflozin treatment group compared to all other groups, (2) the cytokine analysis of the bladder and urethra showed higher IL18 and IL- $1 \beta$ in the dapagliflozin-treated rats and the diabetic rats than in the voglibose-treated rats and the nondiabetic rats, (3) the cytokine levels did not differ between the dapagliflozintreated rats and the diabetic rats, and (4) the level of IL-18 in the dapagliflozin treatment group was higher in the urethra than in the bladder.

Many previous clinical studies and meta-analyses have evaluated the association between UTI and dapagliflozin in patients with T2DM. In a multicenter randomized controlled study, more GIs occurred in the dapagliflozin-treated group $(20 \mathrm{mg} /$ day) than in the placebo group; however, only one patient (4.2\%) in the dapagliflozin $20 \mathrm{mg}$ group reported a UTI [30]. In another multicenter phase III study, though signs and symptoms of GI were more frequent in the dapagliflozin groups $(2.5$ $\mathrm{mg}, 5 \mathrm{mg}$, and $10 \mathrm{mg}$ ) than in the placebo group, UTIs were reported by similar proportions of patients in all groups [31]. Kaku et al. [32] also reported that the signs and symptoms suggestive of UTI were balanced across the treatment groups in a phase II placebo-controlled trial. Additionally, 2 recent metaanalyses showed that SGLT2 inhibitors increased the infection risk for GI, but showed inconsistent results for UTIs [13,20].

As mentioned above, most previous studies have agreed that
SGLT2 inhibitors increase the infection risk for GI, but the UTI-related safety profile of SGLT2 inhibitors remains a concern. Therefore, more detailed information should be provided about the potential mechanisms of action of SGLT2 inhibitors including dapagliflozin for UTIs.

In this study, the 24-hour urine glucose was significantly higher in the OLETF-DA group compared to the other groups, including the OLETF-CO group. Among the multiple potential mechanisms that can contribute to the risk of UTI in persons with diabetes mellitus, a higher urine glucose level may promote the growth of bacteria [6,7]; however in a recent systematic review and meta-analysis of SGLT2 inhibitors and the risk of infections, the authors revealed that other SGLT2 inhibitors, including canagliflozin and empagliflozin, which increase glycosuria, do not appear to increase the risk of UTIs with the exception of high-dose dapagliflozin [13]. Hence, further analyses are required to prove that the high urine glucose concentration is a risk factor for UTI.

As mentioned in the introduction, it is difficult to measure genitourinary infections qualitatively and quantitatively in an animal study. Identifying the difference in inflammatory response of each urinary tract site could be a way to evaluate the mechanism of UTI development indirectly. In addition, to measure the inflammatory response in the dapagliflozin treatment group, we analyzed the concentration of cytokines in the bladder and urethral tissue. Inflammatory cytokines are known to play an important role in mediating the innate immune response, and immune responses are critically important in the 
antimicrobial defense of the urinary tract $[21,22]$. Recently, inflammasomes, which are multiprotein complexes responsible for the activation of inflammatory responses, have emerged as central mediators in the inflammatory bladder most commonly through the maturation and release of the pro-inflammatory cytokines IL-1 $\beta$ and IL-18 [33,34]. IL- $1 \beta$ is one of the first cytokines detected at the onset in infectious cystitis with IL-8, IL-6, and TNFa [35]. Moreover, IL-18 was reported to increase in rat model of cyclophosphamide-induced cystitis [36].

Herein, we found that the level of IL-18 in the urinary tract decreased to the levels observed in the nondiabetic rats in the OLETF-VO group, but remained at the same level as the untreated diabetic rats in the OLETF-DA group, regardless of fasting glucose improvement. In other words, dapagliflozin seems to have more harmful effects on the anti-inflammatory response for UTI than does the comparator antidiabetic drug, voglibose; however, that effect did not show a statistically significant difference compared to the placebo-control, despite a difference in urinary glucose levels.

Our result is also consistent with previous clinical studies in which SGLT2 inhibitors showed more prevalent effects as risk factors for infection in GI than UTI [30-32]. The signs and symptoms suggestive of GI, including vulvovaginitis or balanitis were significantly increased after SGLT2 inhibitor treatment. Urethritis could be closely related to GI because the opening of the urethra is in front of the genitalia. Generally, most cases of UTI including urethritis are characterized by ascending infection. In our experimental setting with male rats, the infection source of urethritis should be genital circumstances. Therefore, the urethritis of a rat can represent GI in experimental setting. Reflecting this assumption, our study showed that the level of IL-18 in the dapagliflozin treatment group was increased 2-fold in the urethra compared to that in the bladder. However, this is just a hypothesis. Researchers should keep in mind that urethritis is not equivalent to GI.

This study has limitations, including the use of dapagliflozin as the only SGLT2 inhibitor. Drug-specific analyses in a recent systematic review and meta-analysis of SGLT2 inhibitors and the risk of infections showed that only $10 \mathrm{mg}$ of dapagliflozin daily was associated with a significantly increased risk of UTI compared to the placebo [13]. Therefore, comparative studies of the anti-inflammatory effects for UTI with other SGLT2 inhibitors, such as canagliflozin and empagliflozin, are needed to identify a specific mechanism of dapagliflozin in UTI. Secondly, this study did not identify microbiological differences among groups as direct evidence of infection. Diverse and numerous bacteria were cultured in all groups and the Enterobacteriaceae were dominant. Unlike clinical trials, aseptic technique is difficult to be applied in animal model. Moreover, it was difficult to determine appropriate parameters to examine statistical significance. Inevitably, microbiological results were excluded from the results. Enterobacteriaceae were the most common organism cultured in this study. On the other hand, the human GI induced by SGLT2 inhibitor is known to be associated with Candida, not Enterobacteriaceae. It can be partially explained that animal model is not equivalent to human body, especially in the genital environment. In order to identify the major microbial causes such as Candida, it is necessary to design careful research in the future.

In conclusion, dapagliflozin, a first-in-class SGLT2 inhibitor, has a demonstrated glucose-lowering effect. This study revealed that dapagliflozin increased the urine glucose concentration, resulting in an inflammatory response remain in the urogenital tract as the untreated diabetic rats. Therefore, when treating patients with T2DM with dapagliflozin, careful attention should be paid to genitourinary infection or inflammation. Further analyses are required to identify whether the high urine glucose concentration is the actual risk factor for genitourinary infection or inflammation. Moreover, pooled comparative clinical or experimental data are required to evaluate the potential risk of SGLT2 inhibitors on genitourinary infection.

\section{AUTHOR CONTRIBUTION}

- Conceptualization: HSC

- Formal Analysis: YJL, SHK

· Investigation: JMY, JWK, SJL, HYK, DSL

- Methodology: YJL, SHK

- Project Administration: SHK, HSC

- Writing - Original Draft: $J B C, H S C$

-Writing - Review \& Editing: JBC, HSC

\section{REFERENCES}

1. Carey IM, Critchley JA, DeWilde S, Harris T, Hosking FJ, Cook DG. Risk of infection in type 1 and type 2 diabetes compared with the general population: a matched cohort study. Diabetes Care 2018;41:513-21.

2. Nitzan O, Elias M, Chazan B, Saliba W. Urinary tract infections in patients with type 2 diabetes mellitus: review of prevalence, diag- 
nosis, and management. Diabetes Metab Syndr Obes 2015;8:12936.

3. Chen SL, Jackson SL, Boyko EJ. Diabetes mellitus and urinary tract infection: epidemiology, pathogenesis and proposed studies in animal models. J Urol 2009;182(6 Suppl):S51-6.

4. Casqueiro J, Casqueiro J, Alves C. Infections in patients with diabetes mellitus: a review of pathogenesis. Indian J Endocrinol Metab 2012;16 Suppl 1(Suppl1):S27-36.

5. Hosking DJ, Bennett T, Hampton JR. Diabetic autonomic neuropathy. Diabetes 1978;27:1043-55.

6. Wang MC, Tseng CC, Wu AB, Lin WH, Teng CH, Yan JJ, et al. Bacterial characteristics and glycemic control in diabetic patients with Escherichia coli urinary tract infection. J Microbiol Immunol Infect 2013;46:24-9.

7. Fünfstück R, Nicolle LE, Hanefeld M, Naber KG. Urinary tract infection in patients with diabetes mellitus. Clin Nephrol 2012;77:408.

8. Rahmoune H, Thompson PW, Ward JM, Smith CD, Hong G, Brown J. Glucose transporters in human renal proximal tubular cells isolated from the urine of patients with non-insulin-dependent diabetes. Diabetes 2005;54:3427-34.

9. Jung $\mathrm{CH}$, Jang JE, Park JY. A novel therapeutic agent for type 2 diabetes mellitus: SGLT2 inhibitor. Diabetes Metab J 2014;38:261-73.

10. Meng W, Ellsworth BA, Nirschl AA, McCann PJ, Patel M, Girotra $\mathrm{RN}$, et al. Discovery of dapagliflozin: a potent, selective renal sodium-dependent glucose cotransporter 2 (SGLT2) inhibitor for the treatment of type 2 diabetes. J Med Chem 2008;51:1145-9.

11. Plosker GL. Dapagliflozin: a review of its use in type 2 diabetes mellitus. Drugs 2012;72:2289-312.

12. Storgaard H, Gluud LL, Bennett C, Grøndahl MF, Christensen MB, Knop FK, et al. Benefits and harms of sodium-glucose co-transporter 2 inhibitors in patients with type 2 diabetes: a systematic review and meta-analysis. PLoS One 2016;11:e0166125.

13. Puckrin R, Saltiel MP, Reynier P, Azoulay L, Yu OHY, Filion KB. SGLT-2 inhibitors and the risk of infections: a systematic review and meta-analysis of randomized controlled trials. Acta Diabetol 2018;55:503-14.

14. Zhang YJ, Han SL, Sun XF, Wang SX, Wang HY, Liu X, et al. Efficacy and safety of empagliflozin for type 2 diabetes mellitus: metaanalysis of randomized controlled trials. Medicine (Baltimore) 2018;97:e12843.

15. Melnick S, Rajagopalan P, Lynn T, Donato A. Perioperative genitourinary infection associated with sodium-glucose co-transporter 2 inhibitor use. J Community Hosp Intern Med Perspect 2018;8:3156.
16. Liu J, Li L, Li S, Jia P, Deng K, Chen W, et al. Effects of SGLT2 inhibitors on UTIs and genital infections in type 2 diabetes mellitus: a systematic review and meta-analysis. Sci Rep 2017;7:2824.

17. Gadzhanova S, Pratt N, Roughead E. Use of SGLT2 inhibitors for diabetes and risk of infection: analysis using general practice records from the NPS MedicineWise MedicineInsight program. Diabetes Res Clin Pract 2017;130:180-5.

18. Li D, Wang T, Shen S, Fang Z, Dong Y, Tang H. Urinary tract and genital infections in patients with type 2 diabetes treated with sodium-glucose co-transporter 2 inhibitors: a meta-analysis of randomized controlled trials. Diabetes Obes Metab 2017;19:348-55.

19. Sano M. Hemodynamic effects of sodium-glucose cotransporter 2 inhibitors. J Clin Med Res 2017;9:457-60.

20. Liu XY, Zhang N, Chen R, Zhao JG, Yu P. Efficacy and safety of sodium-glucose cotransporter 2 inhibitors in type 2 diabetes: a metaanalysis of randomized controlled trials for 1 to 2years. J Diabetes Complications 2015;29:1295-303.

21. Spencer JD, Schwaderer AL, Becknell B, Watson J, Hains DS. The innate immune response during urinary tract infection and pyelonephritis. Pediatr Nephrol 2014;29:1139-49.

22. Zhang JM, An J. Cytokines, inflammation, and pain. Int Anesthesiol Clin 2007;45:27-37.

23. Kawano K, Hirashima T, Mori S, Natori T. OLETF (Otsuka LongEvans Tokushima Fatty) rat: a new NIDDM rat strain. Diabetes Res Clin Pract 1994;24 Suppl:S317-20.

24. Dabhi AS, Bhatt NR, Shah MJ. Voglibose: an alpha glucosidase inhibitor. J Clin Diagn Res 2013;7:3023-7.

25. Han S, Hagan DL, Taylor JR, Xin L, Meng W, Biller SA, et al. Dapagliflozin, a selective SGLT2 inhibitor, improves glucose homeostasis in normal and diabetic rats. Diabetes 2008;57:1723-9.

26. Kim JW, Lee YJ, You YH, Moon MK, Yoon KH, Ahn YB, et al. Effect of sodium-glucose cotransporter 2 inhibitor, empagliflozin, and $\alpha$-glucosidase inhibitor, voglibose, on hepatic steatosis in an animal model of type 2 diabetes. J Cell Biochem 2018 Nov 26:10.1002/jcb.28141. https://doi.org/10.1002/jcb.28141. [Epub].

27. Maurer TS, Ghosh A, Haddish-Berhane N, Sawant-Basak A, Boustany-Kari CM, She L, et al. Pharmacodynamic model of sodium-glucose transporter 2 (SGLT2) inhibition: implications for quantitative translational pharmacology. AAPS J 2011;13:576-84.

28. Staples E, Ingram RJ, Atherton JC, Robinson K. Optimising the quantification of cytokines present at low concentrations in small human mucosal tissue samples using Luminex assays. J Immunol Methods 2013;394:1-9.

29. Mukherjee S, Katki K, Arisi GM, Foresti ML, Shapiro LA. Early TBI-Induced cytokine alterations are similarly detected by two dis- 


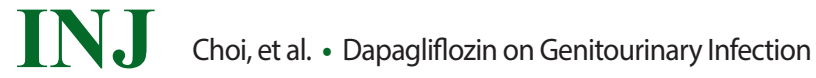

tinct methods of multiplex assay. Front Mol Neurosci 2011;4:21.

30. Wilding JP, Norwood P, T'joen C, Bastien A, List JF, Fiedorek FT. A study of dapagliflozin in patients with type 2 diabetes receiving high doses of insulin plus insulin sensitizers: applicability of a novel insulin-independent treatment. Diabetes Care 2009;32:1656-62.

31. Bailey CJ, Gross JL, Pieters A, Bastien A, List JF. Effect of dapagliflozin in patients with type 2 diabetes who have inadequate glycaemic control with metformin: a randomised, double-blind, placebo-controlled trial. Lancet 2010;375:2223-33.

32. Kaku K, Inoue S, Matsuoka O, Kiyosue A, Azuma H, Hayashi N, et al. Efficacy and safety of dapagliflozin as a monotherapy for type 2 diabetes mellitus in Japanese patients with inadequate glycaemic control: a phase II multicentre, randomized, double-blind, placebocontrolled trial. Diabetes Obes Metab 2013;15:432-40.
33. Martinon F, Burns K, Tschopp J. The inflammasome: a molecular platform triggering activation of inflammatory caspases and processing of proIL-beta. Mol Cell 2002;10:417-26.

34. Purves JT, Hughes FM Jr. Inflammasomes in the urinary tract: a disease-based review. Am J Physiol Renal Physiol 2016;311:F65362.

35. Agace W, Hedges S, Andersson U, Andersson J, Ceska M, Svanborg C. Selective cytokine production by epithelial cells following exposure to Escherichia coli. Infect Immun 1993;61:602-9.

36. Smaldone MC, Vodovotz Y, Tyagi V, Barclay D, Philips BJ, Yoshimura N, et al. Multiplex analysis of urinary cytokine levels in rat model of cyclophosphamide-induced cystitis. Urology 2009;73: 421-6. 